\title{
A pilot study on the functional performance and acceptability of an innovative female condom (Wondaleaf ${ }^{\circledR}$ ) in Malaysia
}

This article was published in the following Dove Press journal:

Open Access Journal of Contraception

\author{
Rachel Sing-Kiat Ting' \\ Ee-Lynn Wong ${ }^{2}$ \\ Jessie Koh-Sing Tnay ${ }^{3}$ \\ 'School of Sociology, China University \\ of Political Sciences and Law, Beijing, \\ China; ${ }^{2}$ InPsych Psychological and \\ Counselling Services, Kuala Lumpur, \\ ${ }^{3}$ Institute of Borneo Studies, \\ Universiti Malaysia Sarawak, Sarawak, \\ Malaysia
}

Background: Female condom (FC) has been available for over 30 years, but it still lacks wide acceptability. To overcome misdirection and invagination occurring in $\mathrm{FC}$ and to provide a wider area of protection, Wondaleaf ${ }^{\circledR}$ (WL), a new-generation adhesive FC, was recently invented. This pioneering study sought to assess the acceptability and functional performance of WL among Malaysian women.

Methods: A mixed method survey was conducted in three cities of Malaysia, recruiting sexually active heterosexual women, aged $18-50$, by snowball sampling method. Participants were provided with WL and initially surveyed to rate its performance in five coital usages over 2 months. After that, the participants underwent a second survey to rate their satisfaction and acceptability toward WL. Descriptive statistics on clinical failure rates were tabulated with correlational analysis performed to identify major variables contributing to WL's functional performance and acceptability.

Results: Out of the 51 enrolled participants, 31 women completed the required surveys. WL's total clinical failure rate was $2.60 \%$ (out of 155 condom uses) with above-average ratings of functional performance. The ease of use significantly correlated with ratings of no slippage and no misdirection. The confidence in WL's safety features significantly correlated with a sense of empowerment and protection.

Conclusion: WL has a relatively low risk of clinical failures and an overall favorable acceptability among Malaysian women. However, this study also showed that its future usage largely depends on partner acceptability. It may have the potential of complementing the existing barrier toward contraceptive use. Further studies are needed to understand the global acceptability of WL.

Keywords: female condom, Wondaleaf, functional performance, acceptability, Malaysian women, contraceptives methods

\section{Background}

Ever since the late 20th century, the creation of the first female condom (FC) by Female Health Company, Chicago, Illinois, USA, has sparked a new movement for "woman initiated" barrier usage under the sexual and reproductive health protection scheme. FCs have enabled women to take preventive measures into their own hands and alleviating men's responsibility for protection. ${ }^{1}$ Like male condoms (MCs), FCs strive to provide dual protection against unplanned pregnancies and most sexually transmitted infections (STIs) including human immunodeficiency virus (HIV). Since year 2000, a variety of FCs have been made readily available to lower the cost and/or improve the acceptability and use of FCs among women. The first-generation FCs have been recognized
Correspondence: Rachel Sing-Kiat Ting Department of Psychology, Jeffrey Cheah School of Medicine and Health Sciences, Monash University Malaysia, Jalan Lagoon Selatan, 47500 Bandar Sunway, Selangor Darul Ehsan, Malaysia

Tel +6084326 631

Email racheltsk@cupl.edu.cn 
as a safe and effective method for preventing HIV and other STIs, and unintended pregnancies among women across cultures. ${ }^{2,3}$ Although often differing in design and materials, newer generation FCs are similar in function. Research has shown that offering both FCs and MCs in reproductive health programs would be the optimal intervention in the prevention of STIs and unintended pregnancies among women. ${ }^{4,5}$ However, FCs are still heavily underutilized, mainly due to sociopolitical reasons and personal preference. ${ }^{6-8}$ As a matter of fact, the utilization of FCs only consists of $0.19 \%$ of the global condom acquisition. ${ }^{8}$

Despite the many benefits of using FCs, including female empowerment, ${ }^{9}$ and it being less dependent on male sexual partner's initative, ${ }^{1}$ feedbacks on FC use are varied. Research showed that the acceptability of FC is often linked to its appearance. ${ }^{10}$ The traditional FC normally is a thin, soft, loose-fitting sheath with a flexible ring placed at the external vagina area and an internal retention unit at the other end. Similar to MC, first, FCs are also being questioned over their protective effect against STIs apart from HIV. ${ }^{4}$ Second, difficulties in the insertion of FCs may deter and influence its usage. ${ }^{11}$ Previous study found that twenty-five percent of the users reported that their first-attempt of FC insertions were difficult and the insertion of retention ring intravaginally may be rather uncomfortable for some women. ${ }^{12}$ Third, though FCs have documented lower risk of breakage than MCs, they faced other problems such as misdirection and invagination due to inexperience of users. ${ }^{13}$ The total clinical failure rate is about $5.24 \%$ for $\mathrm{FC}^{14}$ and below $5 \%$ for newly improved FCs such as FC2 (4.50\%), Cupid2 (4.79\%), and Velvet $(3.93 \%) .{ }^{8}$ Finally, FCs are often more expensive than MCs and can cost up to 2-3 USD per unit in private market, thereby reducing its perceived affordability. ${ }^{15}$ In the past, there were some concerns about the monopoly of FC market due to the limited variety of approved $\mathrm{FCs},{ }^{2}$ and hence there are demands for new FC invention and registration under United States Food and Drug Administration (USFDA) guidelines.

In addressing the aforementioned limitations of both MCs and FCs, an innovative adhesive female barrier film called Wondaleaf (WL) was invented and patented in 2013. WL, manufactured by Twin Catalyst Sdn Bhd in Malaysia, claims itself as the world's first multipurpose female barrier film due to its extensive external adhesive shield. Similar to the conventional condoms, WL aims to prevent unintended pregnancies and STIs, but with additional utilizations for menstrual and female hygiene management. It has also been recently approved by the Malaysian Medical Device Authority (MDA) as a class $\mathrm{C}$ medical device. Although the inventor of WL claims that it could also be used on male population, making it the first unisex condom in the world, this study solely focused on its functional use in women population. Readers could visit www.wondaleaf.com for further details on this product, including its technical structures and usage. This impervious polyurethane FC has an extensive adhesive shield that covers the entire external genitalia, designed to prevent direct skin contact and exchange of body fluid between sexual partners (Figure 1). With a thickness of $20 \mu \mathrm{m}, \mathrm{WL}$ is thinner than the regular latex condom, thereby theoretically improving sensation through better tactile sensitivity and heat transfer. As for internal retention unit, the manufacturer suggests placing a paper tissue or a cotton pad at the tip of the pouch during insertion into the vagina, with application of any water, silicone, or oil-based lubricants. Moreover, WL is designed to rectify the issues of slippage, invagination, and misdirection of conventional FCs, by covering the entire groin with an adhesive external retention system in the form of an extended adhesive film. It also claims to improve the aesthetic look of FCs as it lacks a bulky ring hanging outside of women's body. Apart from its functional characteristics, WL, as a female-controlled method of protection, also claims to be able to give women the much-needed sexual empowerment and to give men greater sexual spontaneity as it could be put on before erection or sexual contact.

In view of the urgent need for more validated and improved FCs in reproductive health and STIs prevention, this study assessed the basic functions of WL as well as its acceptability through a mixed method pilot study among Malaysian women. Effectiveness of contraception and STI prevention of WL is beyond the scope of our study. The first part of this study involved collecting coital logs of WL usage over a period of 8 weeks and the second part of the study assessed the acceptability of WL after five-time usage.

\section{Methods}

\section{Participants}

This study was approved by the ethics committee of School of Sociology at China University of Political Sciences and Law. The inclusion criteria for participants in this study were as follows: 1) women between 18 and 50 years old, 2) sexually active women, 3) sexually healthy women (free from STDs), and 4) women not pregnant at the time of screening. Those who had an untreated/ongoing STI or pregnancy were excluded from the study. The estimated sample size was 50, based on the suggestion for FC clinical investigation study set by $\mathrm{ISO}^{16}$ and previous literatures. ${ }^{17}$ Participants were 


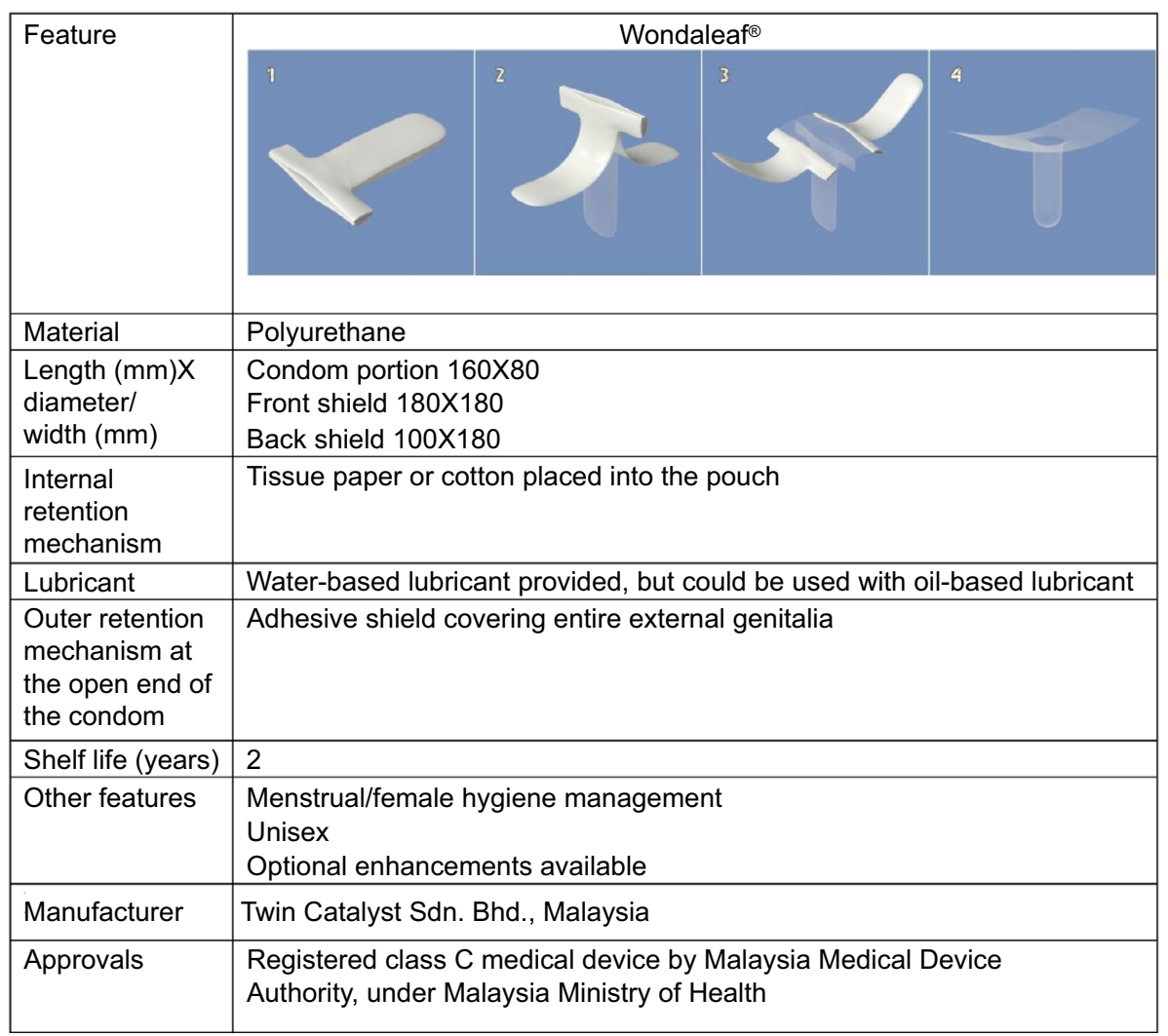

Figure I Basic features of Wondaleaf.

recruited using the snowball sampling method $^{18}$ from various community settings in three different cities of Malaysia. The term "snowball sampling" reflects an analogy to a snowball increasing in size as it rolls downhill. This sampling method depends on the referral of a small pool of initial informants for eligible participants through their social networks, and is commonly used by social sciences researchers for a hardto-reach sample. In our case, snowball sampling method was employed because barrier contraception is not an open topic in Malaysia, so relying on the social network of informants would be more culturally sensitive in the recruitment of participants. Participants were recruited from a university, a medical facility, two family planning clinics, two nongovernmental organizations, and a corporate site.

Due to the cultural sensitivity of the study, participants were enlisted in a private space with a one-on-one discussion with the researchers to ensure confidentiality and to obtain a written informed consent. Throughout the study, participants were encouraged to use other means of contraception (e.g., oral contraceptive pill, intrauterine contraceptive device, and injectable progesterone) in addition to WL to avoid pregnancy. Participants could also request for free pregnancy test kits should they need it at the end of the survey.
Upon signing the informed consent form, each participant was subjected to a standardized training session to ensure proper use of WL. Participants were first asked to view a WL instructional video as well as an in vitro demonstration using a pelvis model. Participants were being guided to practice the donning process of WL using a pelvis model. Upon ensuring participants' competency in using WL, they were provided with six pieces of WL, and cream or lotion should they ask for it. They were asked to use WL five times for sexual intercourse over a 2-month-period, and rate the coital log provided after each use (Part 1 survey). Within 2 months, follow-ups were conducted via email or phone to collect Part 1 survey forms and to distribute Part 2 survey forms. Participants were given 20 Malaysian Ringgit (equivalent to 5 USD) as a token of appreciation upon completion of the study.

\section{Survey instruments}

This study employed a mixed method approach (multimethod research) as both quantitative and qualitative data were collected in our survey. ${ }^{19}$ The survey questionnaires and items were created in consultation with WL's manufacturer, in order to assess the key areas and features of WL. Part 1 survey 
looked into domains of user-friendliness (ease of application and ease of removal) and clinical failure modes (e.g., breakage, slippage, misdirection, and invagination). The adapted operational definitions of clinical failures modes set by the WHO Female Condom Technical Review Committee are as follows: $:^{20,21}$

1. Nonclinical breakage: A breakage, without potential adverse clinical consequences, detected before an intercourse or after the withdrawal of WL from the vagina. ${ }^{20}$

2. Clinical breakage: A breakage, with potential adverse clinical consequences, during sexual intercourse or during withdrawal of WL from the vagina. ${ }^{20}$

3. Slippage: WL that slips out completely from the vagina during sexual intercourse. ${ }^{20}$ Theoretically, complete slippage of WL is unlikely to happen due to its extensive adhesive shield that is attached to the body. However, partial slippage of the non-adhesive portion (pouch) is likely to happen, thus, leaving the definition of slippage open for further investigation in this study.

4. Misdirection: Penis that is inserted in between WL and the vaginal wall, resulting in unprotected vaginal penetration. ${ }^{20}$ Theoretically, misdirection in WL use is impossible, as the external vaginal area is enclosed by the adhesive shield. Misdirection is only possible if the external shield was intentionally removed or broken accidentally. This definition was also open for further investigation in this study.

5 Invagination: WL, inclusive of external retention feature, that is partially or fully being pushed into the vagina during sexual intercourse.

The Part 2 survey covered another five domains of user feedback (i.e., ease of instruction, sensation, empowerment, acceptability, and innovativeness), in which the operational definitions were defined as follows:

1. Ease of instruction: Agreement that a) a step-by-step manual instruction sheet or b) an instructional video in teaching WL usage is easy to comprehend.

2. Sensation: Agreement that there is presence of sensitivity while using WL during coital activity.

3. Empowerment: a) A belief that WL could provide sufficient medical protection for the individual, b) gaining a sense of mastery over one's health from using WL, and c) free of STIs infection concern while using WL.
4. Acceptability: a) Acceptance of WL use by sexual partner, b) willingness to use WL in future, and c) willingness to recommend WL to friends.

5. Innovativeness: Agreeing WL as an innovative product.

In the Part 2 survey, we also designed two open-ended questions for the participants to provide written comments on WL's strengths and areas for improvement. Textual data were further analyzed using qualitative coding method.

All survey questions were simple and direct statements so as to ensure comprehensibility of participants from lower education background. Surveys were available in three languages (Bahasa Malaysia, Mandarin Chinese, and English) to cater to the multilingual community in Malaysia.

\section{Analysis}

All quantitative domains were assessed on a Likert scale ranging from 1 (strongly disagree) to 5 (strongly agree) based on the participants' subjective opinions. We adapted the Likert scale to take into account the individual changes in attitude across five usages. The actual incidences of clinical failure rates of WL (such as slippage, breakage, invagination, and misdirection problems) were confirmed by conducting a post hoc interview among participants who gave unsatisfactory ratings (rating <3) in the coital log. Adopting the definition set by WHO Female Condom Technical Review Committee (2007), total clinical failure for WL is defined as the number of WLs that clinically break or slip, or are associated with misdirection or invagination, during intercourse or any additional failure mode(s) identified in the risk assessment. The total clinical failure rate is calculated by dividing the number of WLs with a clinical failure by the number of WLs used during sexual intercourse. First, descriptive analysis was performed. Next, correlation and regression analyses were performed to further identify factors associated with WL's acceptability. Finally, the written comments from the Part 2 survey were coded using qualitative thematic two-tier coding method, ${ }^{19}$ and the frequency and percentages of the major themes were calculated.

\section{Results}

Data were collected between November 2016 and February 2017. A total of 51 participants were recruited at the beginning of the study. Fifteen participants dropped out of the study, and an adverse event occurred in which one participant became pregnant during the process of the study and her participation was terminated after three trials. While 
36 participants completed both questionnaires (71\% return rate), five incomplete questionnaires were excluded. Only those 31 questionnaires that contained complete details on the five-time use of WL (total 155 condom uses) were included in the result analysis (Table 1). Reasons for the 20 dropout/ incomplete enrollment varied: three were not contactable, eleven withdrew due to the lack of cooperation from their male sexual partner (complaining feelings of dullness or unfamiliarity with using condoms), one had unplanned pregnancy due to ineffective contraception method, two reported limited chances for coital activity over 2-month period (due to poor health or husband being away), one complained WL as not user-friendly, one complained WL as not comfortable, and one had missing data on the returned form. These issues will be further addressed in the Discussion section.

\section{Analysis on technical functions of WL (Part I survey)}

The descriptive analysis showed that most of the items in Part 1 survey were scored between 3 and 5 (Table 2), which suggested participants' agreement on WL's safety features. No incidences of invagination and non-clinical breakage

Table I Participant demographics $(n=3 \mathrm{I})$

\begin{tabular}{lll}
\hline Background data & $\mathbf{n}$ & $\%$ \\
\hline Age (mean) & $34.6 \mathrm{I}$ & 7.6 \\
Ethnicity & 9 & \\
$\quad$ Chinese & 7 & 29 \\
Iban & 5 & 23 \\
Malay & 5 & 16 \\
Bidayuh & 2 & 16 \\
Others & 3 & 6 \\
Not reported & & 10 \\
Highest education level & 4 & \\
Primary school & 2 & 13 \\
PMR & 9 & 6 \\
SPM & 1 & 29 \\
STPM & 11 & 3 \\
University/diploma & 4 & 36 \\
Not reported & & 13 \\
Occupation & 6 & 19 \\
Family planning officer & 6 & 19 \\
Administration officer & 5 & 17 \\
Nurse & 1 & 3 \\
Special needs teacher & 8 & 26 \\
Sex worker & 1 & 3 \\
Sales assistant & 1 & 3 \\
Field laborer & 3 & 10 \\
Not reported & 0 & 0 \\
Previous use of other FC & 5 & \\
Previous use of WL & & \\
\hline Note: PMR sPM, and STPM & & \\
\hline
\end{tabular}

Note: PMR, SPM, and STPM are lower, middle, and upper secondary school respectively.

Abbreviations: FC, female condom; WL, Wondaleaf. occurred throughout the clinical trials, with only one clinical breakage (item 4$)$ occurring during first-time usage $(0.65 \%$, 1 out of 155 condom uses). Items endorsing "no slippage" (item 5) and "no misdirection" (item 6) scored well, with an average rating of 3.8. In the post hoc interview, users described three kinds of "slipping out" conditions that contributed to different clinical connotations. The first being a "feeling of WL sliding inside the vagina, but no actual slipping out from the vagina". The second slippage condition was "occasional slipping of WL's pouch out of the vagina, but it could be reinserted". The third condition identified "a slippage of pouch out of the vagina but not able to be reinserted by the user". In general, none of the reported slippages met the definition of a complete slippage, as the adhesive external retention of WL was still attached to the body. However, as a pilot study, we needed a more conservative definition from the consumers' perspective. Hence, we included the third slippage condition as one of the "clinical failure modes" since WL was discarded under such condition, and the users were exposed to the risk of body fluid exchange. In sum, there were a total of 2 out of 155 condom uses accounting for such high risk slippage, constituting $1.30 \%$ of clinical failures due to slippage ( 2 out of 155 trials).

In the post hoc interview of "no misdirection" (item 6), there were no reports of "penis going in between WL and the vagina"; however, there were incidences of "penis' inability to penetrate the vagina" due to improper placement of WL pouch. Some part of the pouch could get stuck to the external adhesive shield, which resulted in penis sliding outside of vagina. This occurrence of "misdirection" did not fulfill the clinical failure modes defined by $\mathrm{WHO},{ }^{20}$ but this could be categorized as "difficulty in penetration", a potential risk in using adhesive FC. Only one participant removed WL due to "difficulty in penetration", resulting in a failed attempt $(0.65 \%)$.

According to WHO FC guidelines (2012): "As part of the risk assessment, manufacturers shall determine if any additional failure modes may apply to the specific female condom under consideration because of its design, materials of construction or method of manufacture" (p.17). ${ }^{20}$ Because there is no exhaustive definition of what constitutes clinical failures for all FCs, and the existing failure modes might not apply to adhesive condoms like WL, we believe our explorative study can contribute to that by expanding the failure modes of adhesive FCs like WL. After expanding the definition of clinical failures modes by considering the specific features of WL, WL obtained a total clinical failure rate of $2.60 \%$ (Table 2). 
Table 2 Descriptive statistics on Part I and Part 2 survey items

\begin{tabular}{|c|c|c|c|c|c|c|}
\hline \multicolumn{2}{|c|}{ Survey items } & $\begin{array}{l}\text { First use } \\
\text { Mean (SD) }\end{array}$ & $\begin{array}{l}\text { Second use } \\
\text { Mean (SD) }\end{array}$ & $\begin{array}{l}\text { Third use } \\
\text { Mean (SD) }\end{array}$ & $\begin{array}{l}\text { Fourth use } \\
\text { Mean (SD) }\end{array}$ & $\begin{array}{l}\text { Fifth use } \\
\text { Mean (SD) }\end{array}$ \\
\hline \multicolumn{7}{|c|}{ Part I items $(n=3 I)$} \\
\hline I. & Easy to put on WL & $2.39(0.96)$ & $2.58(0.92)$ & $2.97(0.95)$ & $3.32(0.98)$ & $3.39(1.02)$ \\
\hline 2. & Easy to remove $\mathrm{WL}$ & $3.35(1.08)$ & $3.45(0.96)$ & $3.65(0.88)$ & $3.87(0.67)$ & $3.97(0.80)$ \\
\hline 3. & No breakage before use & $4.26(0.68)$ & $4.26(0.68)$ & $4.39(0.58)$ & $4.42(0.56)$ & $4.52(0.57)$ \\
\hline 4. & No breakage after use & $4.03(0.84)$ & $4.03(0.7 I)$ & $4.19(0.54)$ & $4.26(0.58)$ & $4.35(0.61)$ \\
\hline 5. & WL did not slip out during use & $3.32(1.22)$ & $3.48(1.09)$ & $3.71(1.04)$ & $3.97(0.88)$ & $4.03(0.88)$ \\
\hline 6. & Did not miss the direction of penis insertion & $3.68(0.98)$ & $3.74(0.89)$ & $3.94(0.77)$ & $4.10(0.70)$ & $4.10(0.75)$ \\
\hline \multirow[t]{3}{*}{7.} & WL did not get stuck in vagina after use & $4.03(0.48)$ & $4.06(0.5 \mathrm{I})$ & $4.13(0.43)$ & $4.16(0.52)$ & $4.23(0.50)$ \\
\hline & Total & $25.06(4.05)$ & $25.61(3.23)$ & $26.97(2.79)$ & $28.10(2.88)$ & $28.58(3.31)$ \\
\hline & & & Max & Min & Mean & SD \\
\hline \multicolumn{7}{|c|}{ Part 2 items $(n=3 I)$} \\
\hline $\mathrm{I}$. & Manual instruction is easy to follow & & 2 & 5 & 3.97 & 0.71 \\
\hline 2. & Video instruction is easy to understand & & 3 & 5 & 4.42 & 0.72 \\
\hline 3. & $\begin{array}{l}\text { There is enough sensation when using this ba } \\
\text { intercourse }\end{array}$ & during & 1 & 5 & 3.16 & 1.04 \\
\hline 4. & $\begin{array}{l}\text { I believe this barrier film could provide suffic } \\
\text { protection }\end{array}$ & & 2 & 5 & 4.29 & 0.78 \\
\hline 5. & $\begin{array}{l}\text { This female barrier film gives me a sense of } n \\
\text { health }\end{array}$ & ver my & 2 & 5 & 4.16 & 0.90 \\
\hline 6. & $\begin{array}{l}\text { This barrier film could assure my safety in se } \\
\text { worrying of infection }\end{array}$ & ities without & 3 & 5 & 4.42 & 0.67 \\
\hline 7. & My sexual partner could accept this barrier $f$ & & 2 & 4 & 3.16 & 0.82 \\
\hline 8. & I will continue to use this female barrier film & & 1 & 5 & 3.06 & 0.89 \\
\hline 9. & I would recommend this barrier film to $\mathrm{my} \mathrm{fr}$ & & 1 & 5 & 3.87 & 0.81 \\
\hline \multirow[t]{2}{*}{10.} & I found this barrier film innovative & & 2 & 5 & 4.16 & 0.86 \\
\hline & & & \multicolumn{3}{|c|}{ Number of events per total WL used } & $\%$ \\
\hline \multicolumn{7}{|c|}{ Clinical failure modes $(n=\mid 55)$} \\
\hline I. & Clinical breakage & & $1 / 155$ & & & 0.65 \\
\hline 2. & Nonclinical breakage & & $0 / 155$ & & & 0 \\
\hline 3. & Clinical misdirection ${ }^{\mathrm{a}}$ & & $1 / 155$ & & & 0.65 \\
\hline 4. & Nonclinical misdirection ${ }^{\mathrm{b}}$ & & $2 / 155$ & & & 1.30 \\
\hline 5. & Invagination & & $0 / 155$ & & & 0 \\
\hline 6. & Partial slippage ${ }^{c}$ & & $24 / 155$ & & & 15.48 \\
\hline 7. & Complete slippage $^{d}$ & & $2 / 155$ & & & 1.30 \\
\hline 8. & Total clinical failures & & $4 / 155$ & & & 2.60 \\
\hline
\end{tabular}

Notes: I, extremely disagree; 2, disagree; 3, neutral; 4, agree; 5, highly agree. alncorrect vaginal penetration; 'bifficulty in penetration; 'pouch sliding inside the vagina or partially moved outside of the vagina; ${ }^{d}$ the whole FC product detached from human body, either by will or by accident.

Abbreviations: FC, female condom; WL, Wondaleaf.

With regards to user-friendliness, most participants found WL easier to remove than to apply, with an overall mean score of 3.66 (Part 1: item 2) as compared to 2.93 (Part 1: item 1). However, mean scores for ease of application (Part 1: item 1) increased significantly from 2.39 to 3.39 over the course of five usages $(t=5.39, p<0.001)$, with it becoming easier upon three usages. Non-parametric correlation analysis (Table 3) revealed that participants $(\mathrm{n}=31)$ who rated item 1 (ease of application) favorably are more likely to rate item 5 (no slippage) positively (Spearman's $\rho=0.55, p<0.001$ ). Items 5 and 6 (no misdirection) were also significantly correlated (Spearman's $\rho=0.49, p<0.01$ ), implying that the absence of slippage would coincide with lower rates of misdirection. Hence, it
Table 3 Nonparametic correlations matrix between items of Part I $(n=3 \mid)$

\begin{tabular}{llllllll}
\hline Items & Item I & Item 2 & Item 3 & Item 4 & Item 5 & Item 6 & Item 7 \\
\hline I. & 1.000 & $0.30 I$ & -0.087 & 0.109 & $0.548^{* *}$ & $0.25 I$ & 0.306 \\
2. & & 1.000 & -0.215 & -0.026 & -0.077 & -0.059 & $0.396^{*}$ \\
3. & & & 1.000 & $0.67 I^{* *}$ & 0.153 & 0.265 & 0.310 \\
4. & & & & 1.000 & 0.246 & 0.006 & $0.399^{*}$ \\
5. & & & & & 1.000 & $0.487^{* *}$ & 0.342 \\
6. & & & & & & 1.000 & $0.22 I$ \\
7. & & & & & & & 1.000 \\
\hline
\end{tabular}

Notes: $* p<0.05$ (two-tailed); $* *<<0.01$ (two-tailed).

was inferred that participants who found it easier to put on WL would have less issues with slippage and misdirection. 
Age and education level were not significantly correlated with any items in the Part 1 survey.

\section{Analysis on the acceptability toward WL (Part 2 survey)}

Overall, participants were satisfied with WL after five times of usage, as most ratings fell between 4 and 5 , and their attitudes toward future usage (item 8) appeared neutral (Table 2). There also appeared to be a preference for video instruction over manual instruction. A general consensus was found on the confidence over safety features of WL in the protection of STIs and other medical problems (item 6). The participants also agreed to gaining a sense of mastery over their body (item 5) after using WL.

Items 7-9 were significantly correlated with one other (Spearman's $\rho=0.44-0.64, p<0.05$ ) (Table 4). Though most of the participants did not show eagerness in future employment of WL, due to various reasons, 24 out of 31 (77\%) participants would actually recommend WL to their friends. Of note, item 8 ("I would continue to use WL") was significantly correlated with Part 1 item 1 (easy to put on), Part 2 item 3 (sensation), Part 2 item 6 (safety from STD), Part 2 item 7 (acceptability of the partner), Part 2 item 9 (recommending to friends), and Part 2 item 10 (innovativeness). A hierarchical regression on these related variables showed that sensation and the acceptability of their partners were the best predictors for future use of WL $(R=0.763, p<0.001)$.

Finally, 24 out of 31 (77\%) participants concurred WL as an innovative invention (item 10) that was not seen or used previously. This perception was not significantly correlated with either age or education level, but it was significantly correlated with the sense of empowerment (items 4 and 6) and acceptability (items 8 and 9) toward WL (Spearman's $\rho=0.38-0.70, p<0.001)$. In other words, independent of age or educational background, the perception of WL as an innovative product would play its part in the acceptability and belief toward WL.

\section{Qualitative analysis of written feedback}

The qualitative themes on performance of WL were consistent with the quantitative analysis discussed in the previous sections (Table 5). The majority of participants affirmed WL's safety features in protection against STIs and pregnancy. The maintenance of hygiene as well as presence of pleasurable sensations were pointed out as strengths of WL. However, concern with regards to the application of WL were brought forward as some users found it "troublesome" or "inconvenient". The use of tissue paper as retention unit also brought uneasiness to some female participants. The varying opinions given by participants indicated that future usage of WL might be an idiosyncratic preference, as some found it to be practical, while others perceived it as being bothersome. Varying opinions were also observed with regards to sensation, with some strongly endorsing pleasurable sensation while others complained of the lack of sensation during intercourse.

\section{Discussion Clinical performance and failures}

Based on the data gathered, it can be concluded that WL is a safe and functional medical device. Out of the total 155 trials, only four clinical failures $(2.60 \%)$ were observed, setting WL in an encouraging position as compared to previous documented statistics of MCs and FCs. No reports of STIs were observed among 31 participants at the end of the study, though one unintended pregnancy was noted in the dropout sample as adverse event. Compared to past studies on $\mathrm{FCl}$ and FC2, no incidences of invagination occurred with WL in this current study. Its adhesive retention shield makes complete slippage and penetration outside of the condom pouch almost impossible. However, it is probably also due to its adhesive

Table 4 Nonparametic correlations matrix between items of Part $2(n=31)$

\begin{tabular}{|c|c|c|c|c|c|c|c|c|c|c|}
\hline Items & Item I & Item 2 & Item 3 & Item 4 & Item 5 & Item 6 & Item 7 & Item 8 & Item 9 & Item 10 \\
\hline I. & 1.000 & $0.6 \mathrm{I} \mathrm{I}^{* *}$ & -0.054 & 0.053 & 0.077 & 0.027 & 0.285 & 0.023 & 0.056 & -0.064 \\
\hline 2. & & 1.000 & 0.102 & 0.242 & 0.264 & 0.286 & 0.113 & 0.210 & 0.153 & 0.156 \\
\hline 3. & & & 1.000 & 0.185 & 0.247 & $0.357^{*}$ & $0.410 *$ & 0.695 ** & $0.360 *$ & $0.425^{*}$ \\
\hline 4. & & & & 1.000 & $0.727^{* *}$ & $0.632 * *$ & -0.036 & 0.161 & 0.215 & $0.379 *$ \\
\hline 5. & & & & & 1.000 & $0.686 * *$ & 0.100 & 0.275 & 0.219 & 0.309 \\
\hline 6. & & & & & & 1.000 & 0.071 & 0.332 & $0.509 * *$ & $0.628 * *$ \\
\hline 7. & & & & & & & 1.000 & $0.639 * *$ & $0.443 * *$ & $0.413^{*}$ \\
\hline 8. & & & & & & & & 1.000 & $0.624^{* *}$ & $0.534 * *$ \\
\hline 9. & & & & & & & & & 1.000 & $0.701 * *$ \\
\hline 10. & & & & & & & & & & 1.000 \\
\hline
\end{tabular}

Notes: $* p<0.05$ (two-tailed); ${ }^{* *} p<0.01$ (two-tailed). 
Table 5 Thematic coding of written feedback toward WL $(n=3 I)$

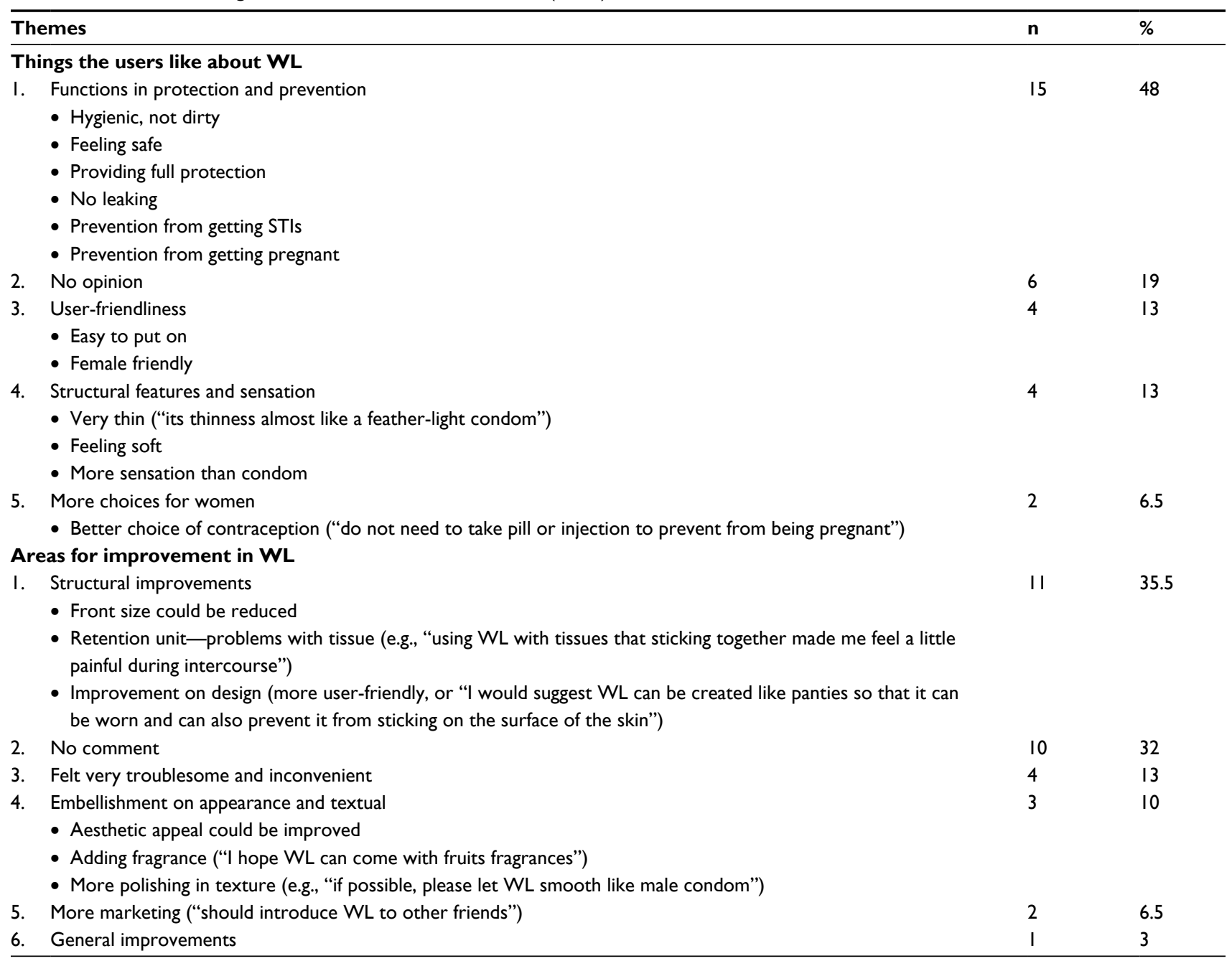

Abbreviations: STI, sexually transmitted infection; WL, Wondaleaf.

nature and ultrathin barrier film that some users found WL difficult to maneuver. Hence, we suggested that the definition of WHO on FC failure modes ${ }^{20}$ be adapted for adhesive FCs such as WL, to better assess its risks (e.g., slipping out of the pouch and difficulty of penetration) and functionality. ${ }^{21}$ There were also no complaints of physical irritation (such as pain, itch, or burning sensations) reported in the survey.

\section{Acceptability factors}

Both subjective and objective ratings from the survey affirmed the perception of safety features of WL and its protection against STIs. This is consistent with WL's claim for dual protection. Participants also agreed that WL is an innovative product that encourages a sense of control over their health and personal hygiene. Yet, most of the users endorsed an impartial opinion on future use. This might probably be due to the demographics of the participants (i.e., 24 out of the 31 participants are monogamous couples) who did not perceive a high risk in STD transmission from their sexual partners. Another factor might pertain to the preference of invisible or non-barrier contraception methods for females living in an Asian culture. Considering the fact that Malaysia is a patriarchal and religious society, almost 30\% (15 participants) of recruited participants dropped out of the study due to disapproval from their male partners. Partner acceptability has always served as a challenge and contending issue in the history of FC proponents. ${ }^{9}$ Our study also showed that WL is not immune to such challenge, even though it has improved the safety features of FC by adopting a larger adhesive shield. The cultural taboo on sex and gender power differentiation in the Asian culture would be the major impediment to the full utilization of WL.

In light of the significant increase of WL's user-friendliness ratings across five trials, this study supported previous FC researchers on the premise that "practice makes perfect". ${ }^{13}$ 
Standardized training is especially critical for WL on its two major steps of application: 1) insertion of the non-adhesive pouch into the vagina and 2) unfolding of the adhesive shield. Improper application of WL could affect its safety and performance as well as acceptability among users. This study also found that participants who had a better understanding of proper application techniques had less trouble with misdirection and slippage, thus presenting with a more positive outlook on WL. Therefore, the researchers suggest that further efforts should be invested in teaching the proper application of WL to users to ensure safer outcomes. A welldesigned training intervention involving insertion practices would increase women's competency, facilitate use of FC, improve their attitudes toward the device, and assist women in successfully negotiating safer sex with their partners. ${ }^{22,23}$

Besides educating women, research showed that it is pertinent for their male partners to have similar knowledge ${ }^{3}$ to increase the acceptability and use of $\mathrm{FCs}^{24}$ As shown in the study, partner acceptability remains a key factor in WL use. Thus, further interventions should also target men to educate and empower them in the acceptance and endorsement of FCs.

\section{Limitations}

First, we acknowledge that the survey instruments had not been previously validated. However, our preliminary results indicated that the sum of Part 1 scores across five usages is significantly correlated to each other. As such, we can ensure the test-retest reliability of the Part 1 survey. Second, it was also identified that items in Part 2 clustered in the predicted domains (user-friendliness, acceptability, empowerment, and attitudes). Hence certain degrees of face and construct validity were obtained in our study. On the other hand, a relatively small sample size $(n=31)$ used for regression analysis should be taken into account due to the limited power. Third, the inconsistency of lubricants used by participants in the study might contribute to the inconsistent performance of WL among users. Fourthly, since we did not use the relationship status (e.g., whether monogamous or not) of participants as one of the screening criteria, or control the use of alternative means of contraception during the study, this study could not be qualified as a clinical trial according to ISO guidelines. The goal of this study instead focused on assessing the risk and safety features of WL as a newly invented FC. Finally, the self-report methods used in this study might involve subjectivity bias in which the participants might have different interpretations of items resulting in different endorsement of ratings. Hence, the addition of a brief structural interview after each use might provide further understanding of the unique challenges in the utilization of WL. Nevertheless, the overall findings of this pilot study remain meaningful and significant in paving the way for future research in this area. Future studies should employ randomized crossover research design, in accordance to ISO and WHO guidelines, to establish WL's dual-protection effectiveness and overall functional performance.

\section{Conclusion}

Decades of research had shown that expanding the options of condom use increase the likelihood of woman finding an acceptable protective method that meets their needs. ${ }^{7}$ Our pilot study found that WL had lower clinical failures than the past FCs records and was generally received well by our Malaysian women participants. Due to its additional safety features, WL could serve as a useful option for women's reproductive health and empowerment. Moreover, the cost of WL is comparable to traditional FCs. However, public endorsements of WL have yet to be established and advocated in both the national and international contexts. Future controlled clinical studies are needed to further determine the utility of WL on a global scale. Furthermore, future studies should focus on WL's efficacy and functional performance for women at risk (e.g., sex workers and women living with HIV-affected partners). As explicated by Peters et al, " "as long as global public policy makers hide behind the argument of high prices or the myth that there is no demand (in FCs) and hence no market, access to female condoms will remain out of reach worldwide". Disputing this myth would fall in the hands of current and future generation health care policy makers and proponents of new-generation FCs.

\section{Acknowledgments}

We are grateful to the following individuals or institutes for their assistance in the recruitment of participants: Matron Fu, InPsych Psychological and Counseling Services, Sarawak Family Planning Association, Federation of Reproductive Health Associations, Malaysia (FRHAM), Sarawak Biodiversity Center, Sarawak Research Society, Rejang Medical Centers, University College of Technology Sarawak, and SinChew Daily Papers. This paper has not been published or submitted simultaneously for publication elsewhere.

\section{Disclosure}

The authors report no conflicts of interests in this work. 


\section{References}

1. Meekers D, Richter K. Factors associated with use of the female condom in Zimbabwe. Int Fam Plan Perspect. 2005;31(1):30-37.

2. Peters A, Jansen W, Van Driel F. The female condom: the international denial of a strong potential. Reprod Health Matters. 2010;18(35): $119-28$.

3. Hoffman S, Mantell J, Exner T, Stein Z. The future of the female condom. Perspect Sex Reprod Health. 2004;36(3):120-126.

4. Macaluso M, Blackwell R, Jamieson DJ, et al. Efficacy of the male latex condom and of the female polyurethane condom as barriers to semen during intercourse: a randomized clinical trial. Am J Epidemiol. 2007;166(1):88-96.

5. Nie L, Liao S, Weeks MR, et al. Promoting female condoms in the sex industry in 4 towns of Southern China: context matters. Sex Transm Dis. 2013;40(3):264-270.

6. Gallo MF, Kilbourne-Brook M, Coffey PS. A review of the effectiveness and acceptability of the female condom for dual protection. Sex Health. 2012;9(1):18-26.

7. Mantell JE, Stein ZA, SusserI. Women in the time of AIDS: barriers, bargains, and benefits. AIDS Educ Prev. 2008;20(2):91-106.

8. Beksinska M, Greener R, Kleinschmidt I, Pillay L, Maphumulo V, Smit J. A randomized non-inferiority crossover controlled trial of the functional performance and safety of new female condoms: an evaluation of the Velvet, Cupid2, and FC2. Contraception. 2015;92(3):261-267.

9. Gollub EL. The female condom: tool for women's empowerment. Am J Public Health. 2000;90(9):1377-81.

10. Cecil H, Pinkerton SD, Bogart LM. Perceived benefits and barriers associated with the female condom among African-American adults. J Health Psychol. 1999;4(2):122-129.

11. Joanis C, Beksinska M, Hart C, Tweedy K, Linda J, Smit J. Three new female condoms: which do South-African women prefer? Contraception. 2011;83(3):248-254.

12. Artz L, Demand M, Pulley LV, Posner SF, Macaluso M. Predictors of difficulty inserting the female condom. Contraception. 2002;65(2):151-157.
13. Beksinska M, Smit J, Joanis C, Hart C. Practice makes perfect: reduction in female condom failures and user problems with short-term experience in a randomized trial. Contraception. 2012;86(2):127-31.

14. Beksinska M, Smit J, Mabude Z, Vijayakumar G, Joanis C. Performance of the reality polyurethane female condom and a synthetic latex prototype: a randomized cross-over trial among South African women. Contraception. 2006;73(4):386-393.

15. United Nations Population Fund (UNFPA). HIV Prevention Gains Momentum. New York: United Nations Population Fund; 2011.

16. ISO-29943-2. Condoms -Guidance on clinical studies - Part 2: female condoms, clinical function studies based on self-reports. 2014. Available from https://www.iso.org/obp/ui/\#iso:std:iso:29943:-2:ed-1:v1:en. Accessed on December 20, 2017.

17. Peters A, van Driel F, Jansen, W. Acceptability of the female condom by sub-Saharan African women: a literature review. Afr J Reprod Health. 2014;18(4):34-44.

18. Morgan DL. The SAGE Encyclopedia of Qualitative Research Methods. Thousand Oaks: SAGE Publications, Inc.; 2008:816-817.

19. Creswell JW, Clark VL. Designing and Conducting Mixed Methods Research. Los Angeles, CA: Sage; 2011.

20. World Health Organization, UNAIDS, UNFPA, Family Health International. Female Condom: Generic Specification, Prequalification and Guidelines for Procurement. Geneva, Switzerland: World Health Organization, UNAIDS, UNFPA, and Family Health International; 2012.

21. Beksinska M, Joanis C, Manning J, et al. Standardized definitions of failure modes for female condoms. Contraception. 2007;75(4):251-255.

22. Choi KH, Hoff C, Gregorich SE, Grinstead O, Gomez C, Hussey W. The efficacy of female condom skills training in HIV risk reduction among women: a randomized controlled trial. Am J Public Health. 2008;98(10):1841-1848.

23. Schuyler C, Masvawure TB, Smit JA, et al. Building young women's knowledge and skills in female condom use: lessons learned from a South African intervention. Health Educ Res. 2016;31(2):260-272.

24. Choi KH, Gregorich S, Anderson K, Grinstead O, Gomez C. Patterns and predictors of female condom use among ethnically diverse women attending family planning clinics. Sex Transm Dis. 2003;30(1):91-8.
Open Access Journal of Contraception

\section{Publish your work in this journal}

Open Access Journal of Contraception is an international, peerreviewed, open access, online journal, publishing original research, reports, reviews and commentaries on all areas of contraception. In addition to clinical research, demographics and health-related aspects, the journal welcomes new findings in animal and preclinical studies

\section{Dovepress}

relating to understanding the biological mechanisms and practical development of new contraceptive agents. The manuscript management system is completely online and includes a very quick and fair peer-review system. Visit http://www.dovepress.com/testimonials.php to read real quotes from published authors. 